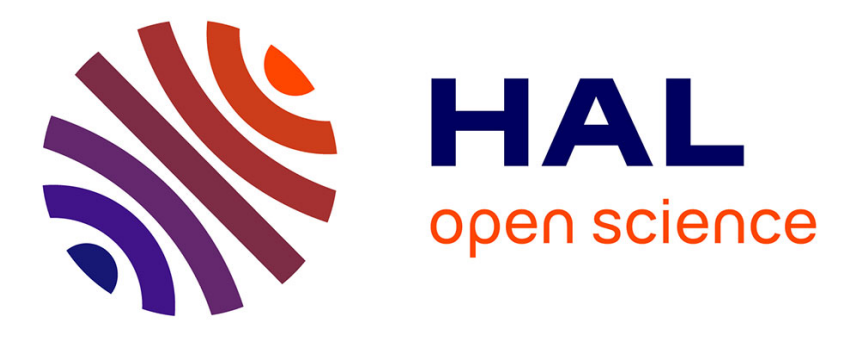

\title{
3D Distribution of Tyrosine Hydroxylase, Vasopressin and Oxytocin Neurons in the Transparent Postnatal Mouse Brain
}

D. Godefroy, C. Dominici, H. Hardin-Pouzet, Y. Anouar, S. Melik-Parsadaniantz, W. Rostène, A. Reaux-Le Goazigo

\section{To cite this version:}

D. Godefroy, C. Dominici, H. Hardin-Pouzet, Y. Anouar, S. Melik-Parsadaniantz, et al.. 3D Distribution of Tyrosine Hydroxylase, Vasopressin and Oxytocin Neurons in the Transparent Postnatal Mouse Brain. Journal of Neuroendocrinology, 2017, 29 (12), pp.e12551. 10.1111/jne.12551 . hal-01628379

\section{HAL Id: hal-01628379 \\ https://hal.sorbonne-universite.fr/hal-01628379}

Submitted on 3 Nov 2017

HAL is a multi-disciplinary open access archive for the deposit and dissemination of scientific research documents, whether they are published or not. The documents may come from teaching and research institutions in France or abroad, or from public or private research centers.
L'archive ouverte pluridisciplinaire HAL, est destinée au dépôt et à la diffusion de documents scientifiques de niveau recherche, publiés ou non, émanant des établissements d'enseignement et de recherche français ou étrangers, des laboratoires publics ou privés. 


\title{
Three-dimensional distribution of tyrosine hydroxylase, vasopressin and oxytocin neurones in the transparent postnatal mouse brain
}

\author{
D. Godefroy ${ }^{1,2}$ | C. Dominici ${ }^{1}$ | H. Hardin-Pouzet ${ }^{3}$ | Y. Anouar ${ }^{2}$ |

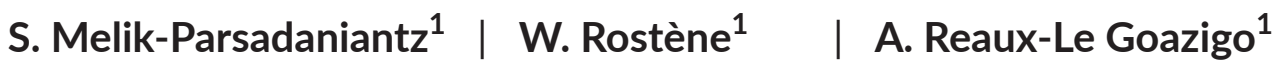

\author{
${ }^{1}$ Institut de la Vision, Sorbonne \\ Universités, INSERM CNRS UMRS 968 \\ UPMC Univ Paris 06, Paris, France \\ ${ }^{2}$ Normandie Université, INSERM, \\ U1239, DC2N, IRIB, UNIROUEN, Mont-Saint- \\ Aignan, France \\ ${ }^{3}$ Neuroscience Paris - Seine Institut \\ de Biologie Paris Seine, Sorbonne \\ Universités, INSERM CNRS, UPMC Univ Paris \\ 06, Paris, France
}

Correspondence

William Rostène, INSERM, CNRS, UMRS 968, Institut de la Vision, Sorbonne Universités, UPMC Univ Paris 06, Paris, France.

Email: william.rostene@inserm.fr

Funding information

The present study was supported by the Université Pierre and Marie Curie. Paris $\mathrm{VI}$, the Institut National de la Santé et de la Recherche Médicale (INSERM) and the Association pour la REcherche sur le Diabète, les Insuffisances cérébrales et le Cancer (AREDIC)

\begin{abstract}
Over the years, advances in immunohistochemistry techniques have been a critical step in detecting and mapping neuromodulatory substances in the central nervous system. The better quality and specificity of primary antibodies, new staining procedures and the spectacular development of imaging technologies have allowed such progress. Very recently, new methods permitting tissue transparency have been successfully used on brain tissues. In the present study, we combined whole-mount immunostaining for tyrosine hydroxylase (TH), oxytocin (OXT) and arginine vasopressin (AVP), with the iDISCO+ clearing method, light-sheet microscopy and semi-automated counting of three-dimensionally-labelled neurones to obtain a (3D) distribution of these neuronal populations in a 5-day postnatal (P5) mouse brain. Segmentation procedure and 3D reconstruction allowed us, with high resolution, to map $\mathrm{TH}$ staining of the various catecholaminergic cell groups and their ascending and descending fibre pathways. We show that TH pathways are present in the whole P5 mouse brain, similar to that observed in the adult rat brain. We also provide new information on the postnatal distribution of OXT and AVP immunoreactive cells in the mouse hypothalamus, and show that, compared to AVP neurones, OXT neurones in the supraoptic (SON) and paraventricular (PVN) nuclei are not yet mature in the early postnatal period. 3D semi-automatic quantitative analysis of the PVN reveals that OXT cell bodies are more numerous than AVP neurones, although their immunoreactive soma have a volume half smaller. More AVP nerve fibres compared to OXT were observed in the PVN and the retrochiasmatic area. In conclusion, the results of the present study demonstrate the utility and the potency of imaging large brain tissues with clearing procedures coupled to novel 3D imaging technologies to study, localise and quantify neurotransmitter substances involved in brain and neuroendocrine functions.
\end{abstract}

KEYWORDS

3D neuronal distribution, catecholamine, iDISCO+, neuropeptide, transparent brain 


\section{1 | INTRODUCTION}

For the past 50 years, immunohistochemical techniques have been recognised to be powerful for the mapping of various neurotransmitter pathways and neuronal cell bodies in the rodent central nervous system (CNS). The development of monoclonal or polyclonal antibodies for various neurotransmitters and neuropeptides enhanced our knowledge of their distribution in various brain regions. In parallel to the immunohistochemical and anterograde or retrograde transport procedures, progress in imaging technologies and computer analyses provided new information concerning neurotransmitter pathways in the CNS. This led to the extensive description of classical neurotransmitters, as well as neuropeptide distributions, in both animal and human brain structures. ${ }^{1,2}$ However, until recently, such tracing approaches relied on tissue sectioning, sometimes followed by serial brain tissue section reconstruction. ${ }^{3}$ These approaches were time-consuming, yielded incomplete spatial distribution and were carried out on thin sections. During the last decade, light scanning microscopy, digital slide scanners and two-photon imaging technologies have improved the detection of fluorescence immunostaining and cell counting on thick sections. However, such methodologies are still limited by the sample size and by light scattering through thick tissues. ${ }^{4}$

New methods of "clearing samples" have emerged recently to avoid such phenomenon, particularly light scattering. ${ }^{4}$ Approaches were also developed to achieve transparency of intact tissues by refractive index homogenisation. ${ }^{4}$ In conjunction with ultramicroscopy, these techniques allowed successful three-dimensional (3D) visualisation. ${ }^{5-7}$ Subsequently, several chemical improvements of the technique "solvent based clearing" and the development of various protocols termed BABB, 3DISCO, iDISCO, iDISCO+ or uDISCO 5,8-13 have been carried out on various tissues. They have provided high-resolution imaging data, which allowed the visualisation of neuroanatomical and neurotransmitter pathways in the brain, trigeminal ganglion or spinal cord of mouse, rat, zebrafish, nonhuman primates or human tissues. ${ }^{10,11,13-17}$

Because extensive studies have been carried out on the distribution of tyrosine hydroxylase (TH), oxytocin (OXT) and arginine vasopressin (AVP) neurones in the rat brain, we have used these neurotransmitter substances to validate the iDISCO+ technique with respect to the mouse brain. We also extended our approach to the generation of 3D maps for these neurotransmitters in the 5-day postnatal (P5) mouse brain by combining whole-mount immunostaining with the iDISCO+ clearing technique and high-resolution ultramicroscopic imaging. These combined methods also permitted a semi-automatic quantitative analysis of the number and volume of specific neuronal AVP and OXT populations in the 3D paraventricular nucleus (PVN).

\section{2 | MATERIALS AND METHODS}

\section{1 | Animals}

Four wild-type adult pregnant female C57BL/6 mice (40 g; Janvier Labs, Le Genest-Saint-Isle, France) were maintained under a
$14: 10$ hours light/dark cycle at $22 \pm 1^{\circ} \mathrm{C}$ and $60 \pm 10 \%$ relative humidity, with access to food and water available ad lib. All animal procedures were performed in accordance with institutional guidelines for the care and use of experimental animals approved by the European Communities Council Directive 2010/63/UE.

\section{2 | Sample preparations}

Twenty-five male and female P5 mice from multiple litters were anaesthetised with a mixture of ketamine $1000 \mathrm{U}\left(80 \mathrm{mg} \mathrm{kg}^{-1}\right.$ body weight) and xylazine ( $8 \mathrm{mg} \mathrm{kg}^{-1}$ bodyweight; Virbac, Carros, France) injected i.p., and brains fixed with an intracardiac perfusion of $4 \%$ paraformaldehyde in phosphate-buffered saline (PBS). Brains were carefully dissected, post-fixed overnight at $4^{\circ} \mathrm{C}$ in the same fixative, and randomly assigned to be immunostained for TH, AVP and OXT.

\subsection{1 | Brain pretreatment with methanol for $\mathrm{TH}$ and OXT immunostaining}

Fixed P5 brains were washed in PBS for 1 hour twice, then in 50\% methanol (in $\mathrm{H}_{2} \mathrm{O}$ ) for 90 minutes, $80 \%$ methanol for 90 minutes and $100 \%$ methanol for 90 minutes. They were then bleached with $3 \%$ $\mathrm{H}_{2} \mathrm{O}_{2}$ in $100 \%$ methanol overnight at $4^{\circ} \mathrm{C}$ in the dark without shaking. Brains were rehydrated in $100 \%$ methanol, $80 \%$ methanol, $50 \%$ methanol, and PBS, allowing 90 minutes for each step before the staining procedures.

\subsection{2 | Brain pretreatment without methanol for AVP immunostaining}

Fixed brains were washed twice each time in PBS for 1 hour and then used for staining procedures.

\subsection{Whole mount staining for TH, OXT and AVP}

Pretreated brains were incubated for blocking and permeabilisation in PBSG-T (PBS, $0.2 \%$ gelatin and $0.5 \%$ Triton- $\mathrm{X} 100$ ) containing thimerosal $\left(0.1 \mathrm{~g} \mathrm{~L}^{-1}\right)$ for 2 days at $37^{\circ} \mathrm{C}$ under agitation, and then incubated with previously characterised primary antibodies (rabbit TH; Millipore, Guyancourt, France; \#AB152, dilution 1:300), ${ }^{10}$ rabbit OXT (immunogen: Cys-Tyr-Ile-Gln-Asn-Cys-Pro-Leu-Gly-NH2; Phoenix Pharma Inc., Strasbourg, France; \#G-051-01, dilution 1:250) and rabbit AVP (immunogen: Cys-Tyr-Phe-Gln-Asn-Cys-Pro-Arg-Gly-NH2, disulfide bridge: Cys1-Cys6; Phoenix Pharma Inc.; \#G-065-07, dilution 1:500) in PBSG-T containing $0.1 \%$ saponin for 7 days at $37^{\circ} \mathrm{C}$ with gentle shaking. The primary antibodies for neuropeptides did not cross-react with each other, nor did they cross-react with other hypothalamic peptides such as growth hormone peptide, gonadotrophin-releasing hormone, thyrotrophin-releasing hormone, vasoactive intestinal peptide, $\alpha$-atrial natriuretic peptide and met-enkephalin as provided by the manufacturer. Brains were washed in PBS for 1 day and incubated with a secondary antibody (donkey anti-rabbit Cy3; Jackson ImmunoResearch, Newmarket, UK; \#705-165-147, dilution 1:500) and TO-PRO 3 iodide 
(Invitrogen, Villebon, France; dilution 1:80) in PBSG-T containing 0.1\% saponin for 2 days at $37^{\circ} \mathrm{C}$ in a dark room with gentle shaking. Finally, they were washed in PBS for 36 hours before the clearing procedure.

\subsection{Clearing procedure}

P5 brains were cleared with the following iDISCO+ protocol. ${ }^{10}$ They were dehydrated in 20\%, 40\%, 60\% and $80 \%$ methanol in $\mathrm{H}_{2} \mathrm{O} 1$ hour for each step and $100 \%$ methanol for 1 hour twice. Brains were incubated overnight in one volume of $100 \%$ methanol/2 volumes $100 \%$ dichloromethane anhydrous (\#DCM, 270997; Sigma-Aldrich, St Quentin-Fallavier, France) and washed twice for 20 minutes in 100\% DCM. Finally, brains were incubated in 100\% DiBenzyl Ether (DBE; \#108014; Sigma-Aldrich) for at least 3 hours before imaging.

\section{5 | Light-sheet imaging}

Cleared P5 brains were imaged with an Ultramicroscope (LaVision BioTec, Bielefeld, Germany) using IMSPECTORPRo (LaVision BioTec). The light sheet was generated by a laser (wavelength 561 nm or 640 nm; Coherent Sapphire Laser; LaVision BioTec) and two cylindrical lenses. A binocular stereomicroscope (MXV10; Olympus, Tokyo, Japan) with a $2 \times$ objective (MVPLAPO; Olympus) was used at different magnifications $(0.63 \times, 3.2 \times, 4 \times, 5 \times)$. Brains were placed in a horizontal or coronal orientation in an imaging reservoir made of $100 \%$ quartz (LaVision BioTec) filled with DBE and illuminated from the side by the laser light. Images were acquired with a PCO Edge SCMOS CCD Camera $(2560 \times 2160$ pixel size; LaVision BioTec). The step size in Z-orientation between each image was fixed at $2 \mu \mathrm{m}$ resolution for $1.26 \times$ magnification and $1 \mu \mathrm{m}$ for $6.4 \times, 8 \times$ and $10 \times$ magnifications, respectively.

\section{6 | Image processing, cell counting and neuronal cell volume determination}

Images of the CNS were constructed from a Z-series of ultramicroscope fluorescence images using IMARIS, version 8.3.0_64 (http:// bitplane.com). Option software tools in IMARIS were used for 3D reconstruction and segmentation from a juxtaposition of serial two-dimensional images of the region of interest according to the mouse brain atlas of Franklin and Paxinos. ${ }^{18}$ False colours were given to these brain areas with IMARIS. High-quality 3D images were selected for neuronal cell quantification and cell volume analysis in the PVN.

Cell counting was conducted for three animals per condition in the magnocellular, medial parvocellular and ventral parvocellular subdivisions of the PVN. Each PVN subdivision was identified by its anatomical localisation.

For accurate AVP and OXT expressing cell automatic counting, IMARIS parameters such as the minimum diameter of the cells $(12.0 \mu \mathrm{m}$ for AVP neurones; $6.5 \mu \mathrm{m}$ for OXT neurones), as well as the threshold of the fluorescence, were set. After confirming that all nerve cells were detected, possible false positive cells were discarded to ensure that each cell was outlined properly.
For cell volume measurements, the quantification was made in the magnocellular subdivision of the PVN. Briefly, following the elimination of the background (surface grain size: $2.50 \mu \mathrm{m}$; diameter of largest sphere: $4.88 \mu \mathrm{m}$ ), the fluorescence thresholding and the minimum number of voxels ("number of voxels" above 1090 to AVP and 900 to OXT) were set, allowing false positives to be discarded and the volume (in $\mu \mathrm{m}^{3}$ ) of the selected neuronal cell bodies to be measured on both sides of the PVN.

$3 \mathrm{D}$ pictures and tiff series were obtained using the optional «animation» tools. Movies were generated with FIJ (NIH, Bethesda, MD, USA). Titles and transition additions were performed using MOVIE MAKER (Microsoft Corp., Redmond, WA, USA).

\section{7 | Statistical analysis}

Data are expressed as the mean \pm SEM and analysed with PRISM, version 7 (GraphPad, San Diego, CA, USA). The difference between AVP and OXT cell volumes was determined using the Mann-Whitney test and that for cell counting using an unpaired $t$ test. $P<.05$ was considered statistically significant.

\section{3 | RESULTS}

\section{1 | 3D mapping of TH neurones with the iDISCO+ technique in P5 mouse brain}

TH immunostaining was first used to demonstrate the robustness of the iDISCO+ methodology by determining the various catecholaminergic structures and pathways present in the whole P5 postnatal mouse brain. In the present study, we delineated and tracked $\mathrm{TH}$ staining in $3 \mathrm{D}$ using the original nomenclature $\mathrm{A} 1$ to $\mathrm{A} 16$ of the various catecholamine-containing cell groups, as initially described by Anden et al. ${ }^{19}$ and Hökfelt et al. ${ }^{20}$ Following the whole mount labelling procedure with a highly specific anti-TH antibody and advanced image processing, a pronounced $\mathrm{TH}$ immunoreactivity corresponding to the A1-A16 cell groups was detected in a rostrocaudal localisation (Figure 1A), with the most rostral A16 dopamine cells located in the olfactory bulb (white); strong dopamine staining in the striatum and anterior cortical areas (blue) (Figures 1B,C and 2A); and catecholaminergic (dopamine, noradrenaline, adrenaline) innervation of the hypothalamus (green) (A11-A15) (Figure 1B,C). In the mesencephalon, a clear distinction was delineated between the well-known $\mathrm{A} 10$ dopamine neurones in the ventral tegmental area (blue) (Figure 1B,C and 2A) corresponding to the ventromedial dopaminergic pathways projecting rostrally to the nucleus accumbens and the frontal cortex, and the nigrostriatal pathway of the A9 dopaminergic neurones (Figure 1B,C, 2A). Finally, caudal to the A9 dopamine neurones in the substantia nigra, some cells (A8) dorsal to the medial and lateral lemniscus were detected, as well as strong noradrenergic staining in the raphe nuclei (Figure 1B,C; see also Supporting information, Movie S1).

Regarding the adrenergic/noradrenergic neurones located in the pons (yellow, Figure 1B) and in the medulla oblongata (pink), the A1-A6 

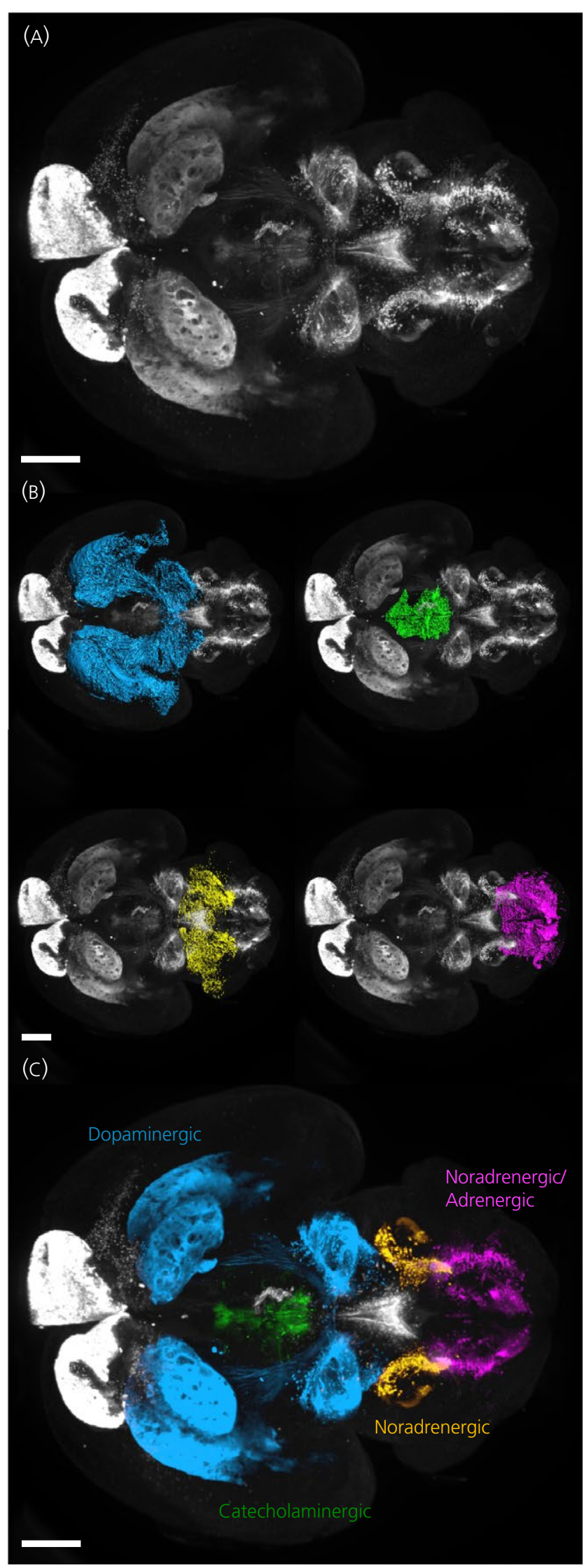

groups of noradrenergic cell bodies were obsevred. More caudally, the two groups of adrenergic/noradrenergic cells were found in the nucleus
FIGURE 1 Five-day postnatal (P5) mouse brain stained with an anti-tyrosine hydroxylase antibody. (A) Three-dimensional horizontal view of the P5 mouse brain. (B) Segmentation and 'surface' rendering used to apply the false colours to the original image of the various anatomical regions with IMARIS, version 8.3.0: telencephalon (blue), hypothalamus (green), pons (yellow) and medulla (purple). (C) Attribution of false colours and 'volume' rendering using for the main catecholaminergic populations: dopaminergic (blue), various catecholamines (green), noradrenergic (white and orange) and noradrenergic/adrenergic (purple) neurones. Magnification 1.26x. Scale bar $=1000 \mu \mathrm{m}$

the solitary tract (A2/C2) and in the ventral medulla/facial nucleus (A1/ C1) (Figure 2; see also Supporting information, Movie S2).

The ascending and descending catecholaminergic pathways innervating the various parts of the brain, particularly the hypothalamus, were visualised using the iDISCO+ technique (Figure 2B; see also Supporting information, Movie S2).

From optical coronal sections, IMARIS was able to reconstruct, in false colours, the 3D TH innervation in defined structures such as the catecholaminergic system in the hypothalamus (Figure 3; see also Supporting information, Movie S1). Specific TH immunoreactive cells were mainly found in the anteroventral periventricular nucleus, around the third ventricle in the periventricular region, and in the most ventromedial part of the suprachiasmatic nucleus. TH immunoreactive neurones extended caudally to the posterior hypothalamus (A14 neurones in cyan). The A11-A13 dopamine neurones (in green) were visualised in the dorsal hypothalamus/zona incerta, as were the $\mathrm{A} 12$ neurones (purple), which constitute the main infundibular dopaminergic neurones in the mediobasal hypothalamus, arcuate nucleus and median eminence (Figure 3; see also Supporting information, Movie S1).

\section{2 | 3D mapping of AVP and OXT immunoreactive neurones in P5 mouse hypothalamus}

By contrast to the rat brain, particularly during the postnatal period, very few studies have investigated the distribution of both neuropeptides AVP and OXT in the mouse brain. Therefore, we used the potent iDISCO+ technique to obtain new information about the distribution of these neuropeptides in the P5 mouse hypothalamus. AVP and OXT are known to be mainly expressed in magnocellular and parvocellular neurones in the PVN and in the supraoptic nucleus (SON), as well as in a number of scattered hypothalamic and contiguous regions so-called accessory nuclei. ${ }^{21-24}$

We confirmed, in P5 brain with the iDISCO+ clearing technique, the well-known distribution of AVP and OXT neurones as described in the adult hypothalamus, the presence of AVP neurones in the suprachiasmatic nucleus and both AVP and OXT immunostaining in accessory nuclei (Figures 4 and 5). Bands of immunoreactive cells were also observed ventrally on both sides of the median eminence, extending far anterior through the medial tuberal nucleus to the retrochiasmatic area. These cells appeared to be more 


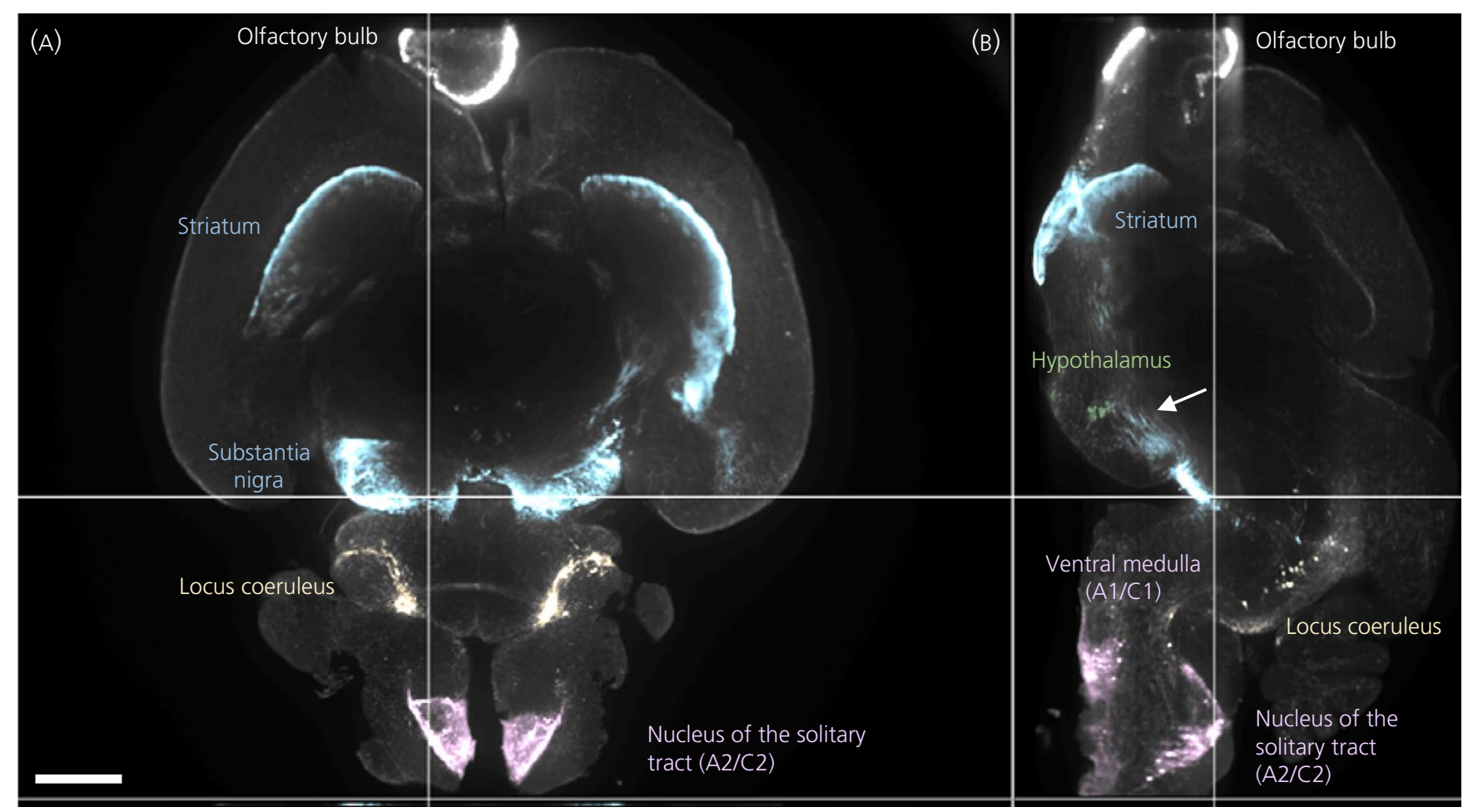

FIGURE 2 Optical horizontal section $(2 \mu \mathrm{m})$ of 5-day postnatal mouse brain stained with an anti-tyrosine hydroxylase (TH) antibody and segmentation of the various groups of catecholamine neurones with IMARIS, version 8.3.0 (A). TH positive neurones in the olfactory bulb (white), dopaminergic neurones in the striatum, substantia nigra (A9) and ventral tegmental area (A10) (in blue); noradrenergic neurones mainly detected in the pons in the locus coeruleus (A6) (yellow) and adrenergic/noradrenergic neurones (purple) in the medulla oblonglata (A1/C1) and in the nucleus of the solitary tract (A2/C2). (B) Optical sagittal section $(2 \mu \mathrm{m})$ of the mouse brain showing some TH positive fibre pathways (arrow) innervating the hypothalamus (green). The sagittal section in (B) corresponds to the vertical bar in (A). Magnification $1.26 \times$. Scale bars $=1000 \mu \mathrm{m}$

FIGURE 3 Five-day postnatal mouse hypothalamus stained with an anti-tyrosine hydroxylase antibody. (A) Optical coronal sections $(2 \mu \mathrm{m})$ at the level of the anteroventral periventricular nucleus, the periventricular region and the dorsal/mediobasal hypothalamus. Scale bar $=200 \mu \mathrm{m}$. (B) Three-dimensional segmentation of the anatomical structures with IMARIS, version 8.3.0, representing the dopaminergic neurones in the dorsal hypothalamus and zona incerta A11-A13 (green), arcuate nucleus $\mathrm{A} 12$ (purple) and in the periventricular $\mathrm{A} 14$ region (cyan). TO-PRO 3 iodide staining (glow) was used to label all nuclei. Magnification 6.4×. Scale bar $=300 \mu \mathrm{m}$

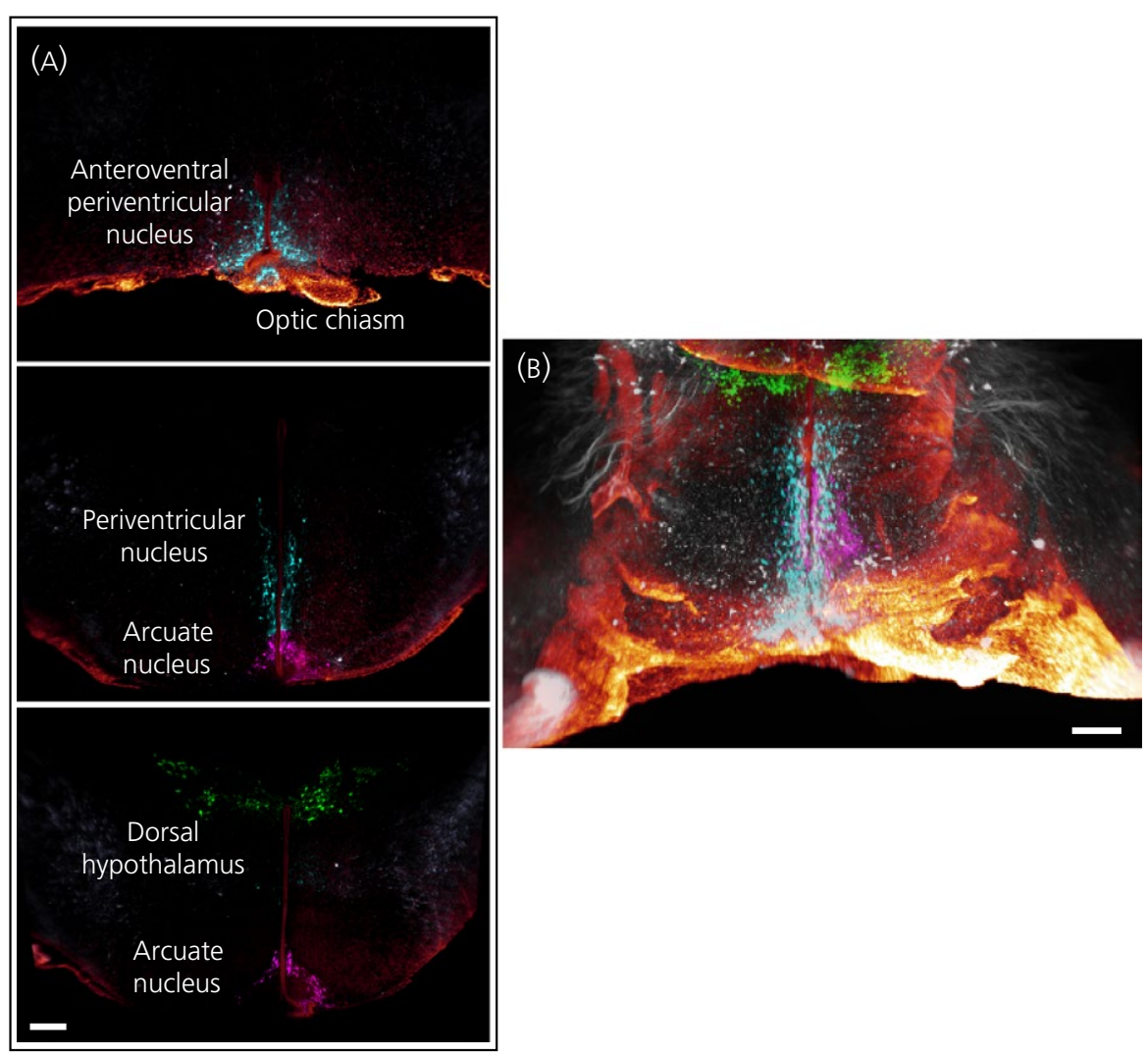



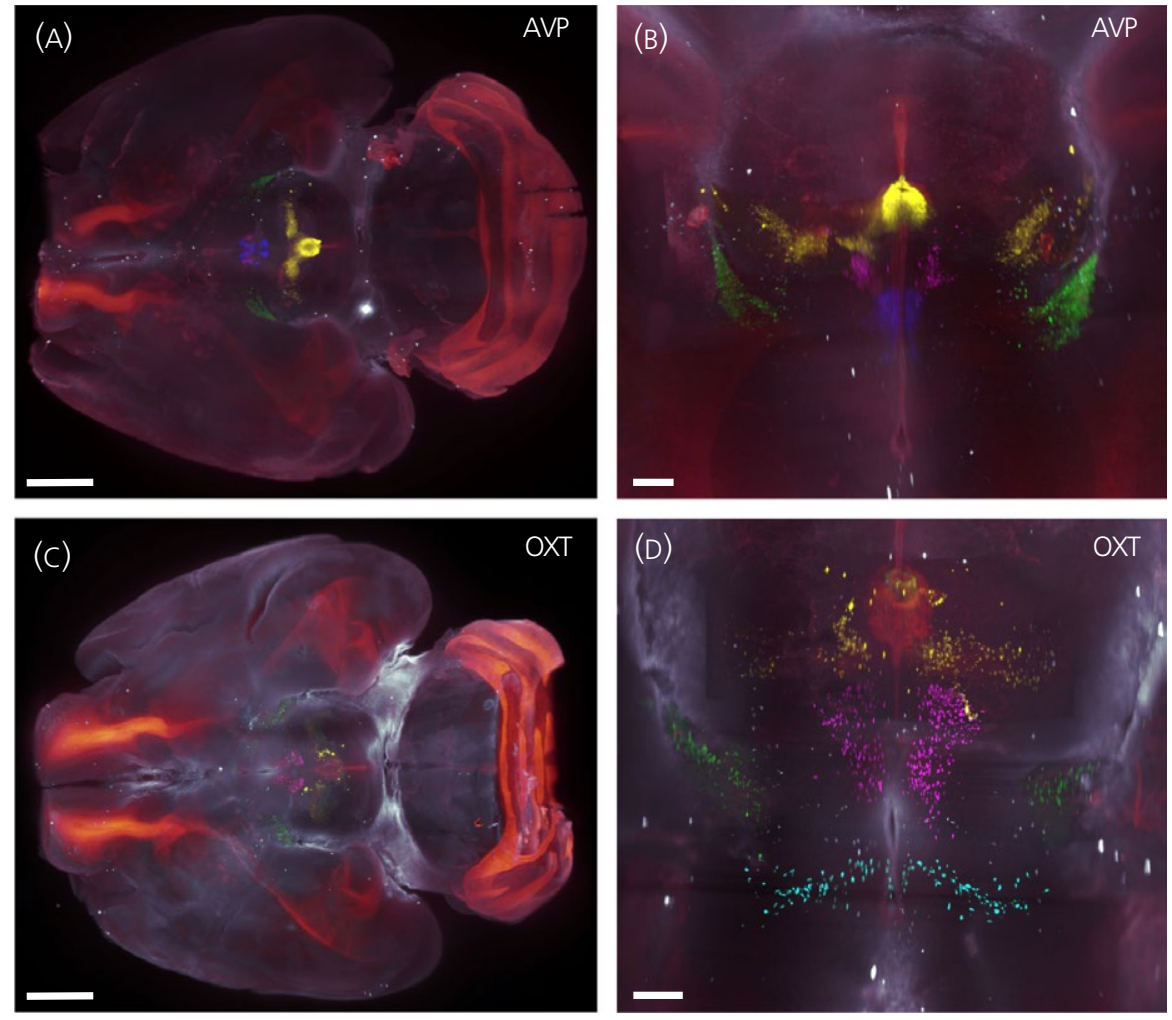

FIGURE 4 Three-dimensional optical horizontal sections of 5-day postnatal mouse brain stained with an anti-arginine vasopressin (AVP) antibody (A) and an anti-oxytocin (OXT) antibody (C). Similar optical coronal views with an anti-AVP (B) and anti-OXT antibodies (D). Supraoptic nucleus (green), paraventricular nucleus (purple), suprachiasmatic nucleus (blue), accessory neurones (cyan) and mediobasal hypothalamus (yellow). TO-PRO 3 iodide staining (glow) was used to label all nuclei. (A, C) Magnification 1.6×, scale bars $=1000 \mu \mathrm{m}$. (B, D) Magnification 6.4x. Scale bars $=200 \mu \mathrm{m}$ numerous for AVP than OXT and had a different distribution pattern (Figure 5C,D; see also Supporting information, Movies S3 and S4).

3D images also clearly show the different labelling of AVP and OXT neuronal cell bodies within the PVN at P5. Indeed, the intensity of AVP immunoreactivity was higher in the magnocellular part of the PVN than in the medial and ventral subdivisions of the parvocellular part of the nucleus (Figure 5A). By contrast, at that age, the fluorescence intensity of OXT immunoreactive neurones was similar in the whole PVN (magno- and parvocellular parts) (Figure 5B; see also Supporting information, Movie S4).

The high-quality images allowed us to perform a semi-automatic counting of AVP and OXT immunoreactive cell bodies throughout the entire 3D PVN. Our results show that PVN at P5 contains approximately three times more OXT cells than AVP neurones (1221 vs 409 neuronal cells, respectively). Semi-automatic counting detected $622 \pm 11$ OXT cells vs $152 \pm 6$ AVP magnocellular neurones $(P<.01 ; n=3$ each condition), $528 \pm 3$ vs $145 \pm 2$ in the medial parvocellular part of the PVN $(P<.01 ; n=3$ each condition $)$ and $71 \pm 4$ vs $107 \pm 2$ in the most ventral parvocellular subdivision of the PVN at P5 ( $P<.01 ; \mathrm{n}=3$ each condition) (Figure 6$)$. In addition, at that age, the volume of the soma of the OXT cells was much smaller than that of AVP neurones (Figure 6; see also Supporting information, Movies S3 and S4). Cell volumes measured on 3D images showed that magnocellular AVP neurones were significantly bigger than the magnocellular OXT neurones in the dorsal part of the PVN : AVP neurones: $1878 \pm 181 \mu \mathrm{m}^{3}$ vs OXT neurones: $1172 \pm 47 \mu \mathrm{m}^{3}(\mathrm{n}=3$ each condition; $P<.01$ ).

\section{4 | DISCUSSION}

The present study was carried out aiming to demonstrate the utility of the iDISCO+ novel clearing technology with respect to mapping phenotypically-identified neuronal cell bodies and pathways in nontransgenic postnatal mouse brain, in particular in the hypothalamus. $\mathrm{TH}$ staining was first used to map catecholaminergic neuronal cells and nerve fibres, demonstrating that enzymes can be targeted using such techniques in mouse brain. Interestingly, the present study also demonstrates that this technique is not restricted to the detection of high molecular weight proteins because we were also able to map nine amino acids neuropeptides (AVP and OXT) with the iDISCO+ clearing technique described here.

Comparing the precision of the present data with other reports using CLARITY, it appears that the iDISCO+ approach provides new information on the $3 D$ organisation of immunoreactive neuropeptide distribution and offers new possibilities for studying multiple cell populations without the necessity of using genetically modified animals expressing various fluorescent proteins. ${ }^{10,11,25,26}$

The 3DISCO technique, first described by Dodt et al., ${ }^{6}$ has recently been extensively improved by means of various solvent solutions that increased the clearing process and decreased light scattering, which hampered the image quality. $8,9,25$ Thus, in the present study, we were able to decrease the tissue shrinkage and obtain a better clearing of the brains by means of methanol treatment instead of tetrahydrofurane as used previously. ${ }^{12,13}$ The clearing procedure did not modify the size of the brain tissue. ${ }^{10}$ However, the various chemical treatments of the clearing procedures could alter antibody specific recognition of the 

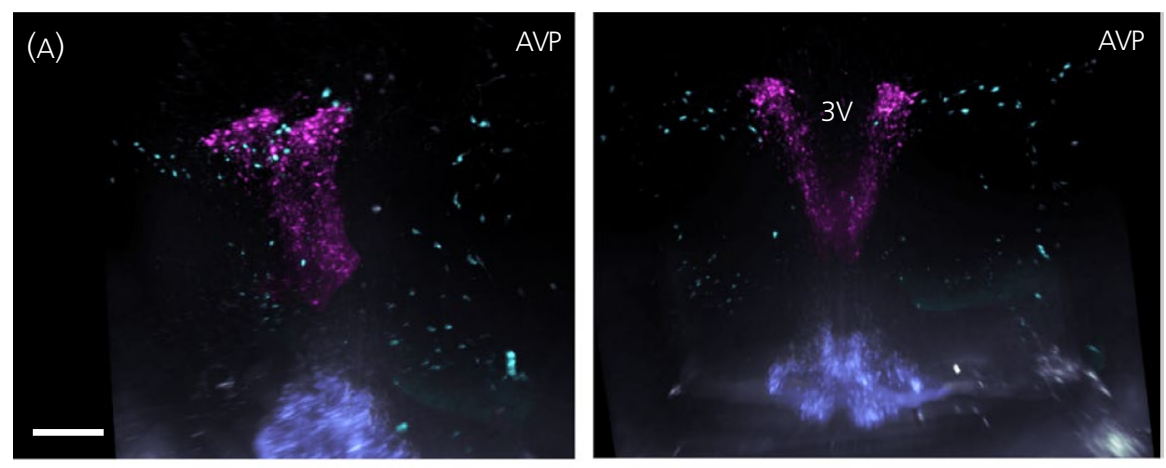

FIGURE 5 Three-dimensional imaging of 5-day postnatal mouse paraventricular nucleus $(A, B)$ and half anterior hypothalamus $(C, D)$ immunostained with anti-arginine vasopressin (AVP) $(\mathrm{A}, \mathrm{C})$ and anti-oxytocin (OXT) (B,D) antibodies. Segmentation of the suprachiasmatic nucleus (blue), supraoptic nucleus (green), paraventricular nucleus (purple), accessory neurones (cyan) and median eminence area (yellow) with IMARIS, version 8.3.0. (A,B) Magnification 10×, scale bars $=200 \mu \mathrm{m}$. (C, D) Magnification $8 \times$, scale bars $=300 \mu \mathrm{m} .3 \mathrm{~V}$, third ventricle
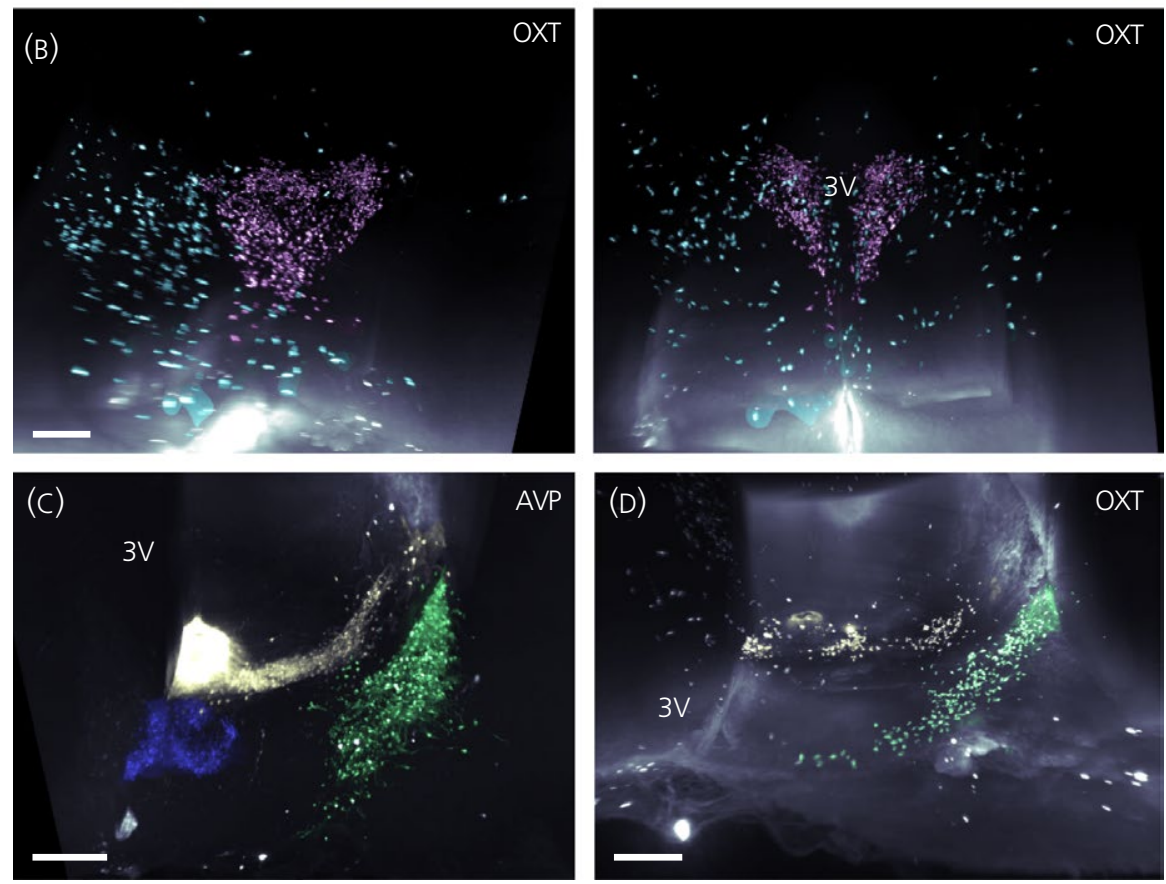

epitope. For example, we observed that some specific polyclonal antibodies against AVP, previously used on brain sections, did not work properly with the methanol procedure (not shown). To avoid this shortcoming, we have used purified antibodies for which the specificity was previously validated by classical immunohistological procedures. Another limitation has been reported concerning the presence of more lipids in adult brains compared to young brains, which may hamper the immunostaining process of some antibodies. ${ }^{10}$

Despite these few limitations, the methodology using light-sheet microscopy, as described in the present study, provides a highresolution 3D imaging of intact brains. Segmentation of the staining with false colours allowed us to map, distinguish and identify the different catecholaminergic systems present in/or innervating various brain areas of the P5 mouse. The iDISCO+ approach of imaging large tissues microscopically at different magnifications provides quantitative information on the morphology, number and volume of cells, as well as information about their projections in the brain. ${ }^{11,16}$ The robustness of the technique was demonstrated in the present study when comparing the results obtained with the reference works related to the various types of catecholaminergic cell groups. ${ }^{20,27-31}$ Here, each of them (dopaminergic, noradrenergic and adrenergic cells) has been determined independently, and some of their ascending and descending pathways were followed by 3D imaging (Figures 2 and 3). Moreover, such catecholaminergic pathways are similar to what is known for the adult rat brain. ${ }^{1,20}$ Similarly, TH immunoreactive localisation such as that previously shown in the rat brain during ontogenesis was seen in the whole P5 mouse brain and especially in the hypothalamus. ${ }^{31-36}$

In both the SON and PVN, especially in magnocellular cells, the main $\mathrm{TH}$ innervation was represented by noradrenergic pathways originating from the brainstem. ${ }^{30}$ Even at P5, as in adults, varicosities on axons such as in TH-expressing anteroventral periventricular neurones and periventricular neurones could be observed (see Supporting information, Movie S1). Finally, we confirmed what was reported in the rat hypothalamus during ontogenesis, ${ }^{31}$ and also in the adult, ${ }^{20}$ in terms of catecholaminergic neurones giving rise to two different populations localised ventrally, mainly in the arcuate nucleus (A12), and in the zona incerta (A11-A13) in the dorsal hypothalamus (Figure 3). It was observed that dopaminergic neurones present in the adult arcuate nucleus had sizes similar to those found in fetuses, whereas those in the dorsal hypothalamus increased in size, forming ramified processes during ontogenesis. ${ }^{31}$ Observations over the lifespan were not the aim 

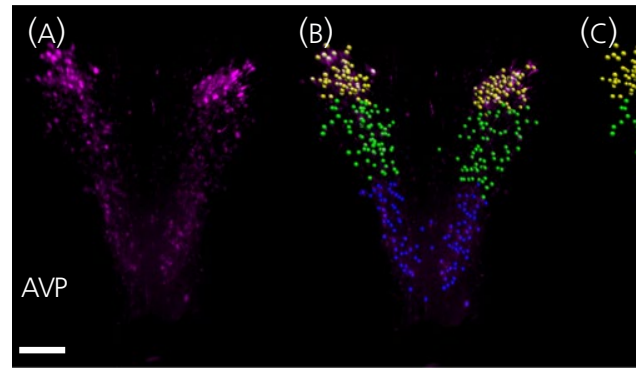

(E)

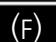 \\ (F)}
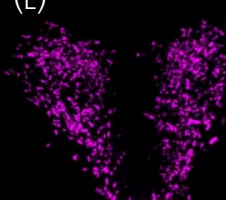

है:
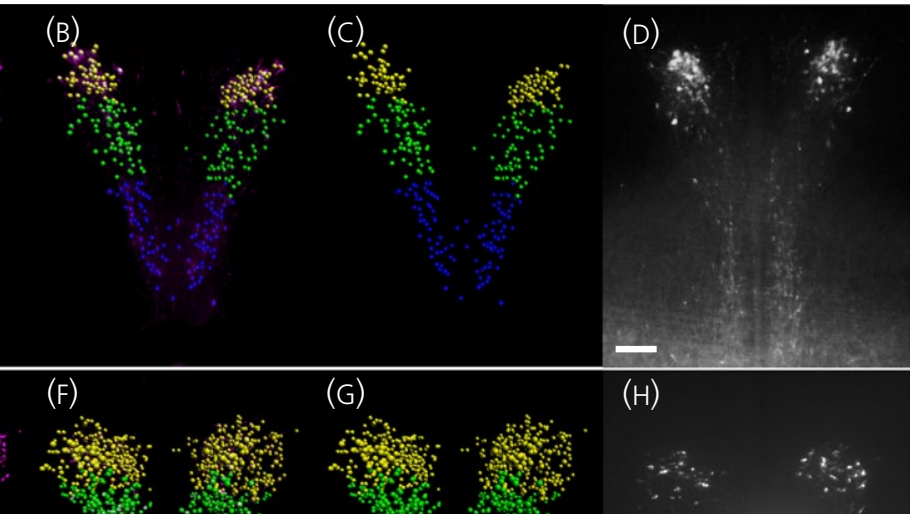

(H)

OXT

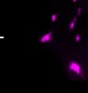

$\rightarrow$
(G)

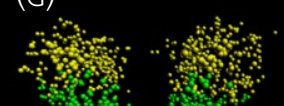

$-4$

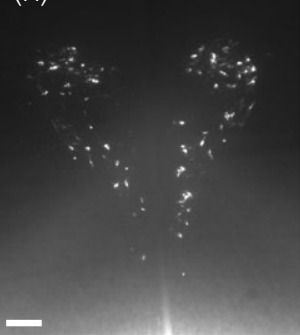

FIGURE 6 Segmentation and semi-automatic counting with IMARIS, version 8.3.0, of arginine vasopressin (AVP) (A-C) and oxytocin (OXT) (E-G) neurones in the whole paraventricular nucleus (purple) and specifically in its magnocellular (yellow), medial (green) and ventral (blue) parvocellular parts in 5-day postnatal mouse hypothalamus $(n=3$ each condition). ( $D, H$ ) Single $1 \mu \mathrm{m}$ deep optical coronal sections at the level of the paraventricular nucleus showing the neuritic processes for both AVP (D) and OXT $(H)$ neurones. Magnification 10x. Scale bars $=100 \mu \mathrm{m}$

of the present study, although such detailed analyses could be performed in future studies using the iDISCO+ method.

The neurohormones AVP and OXT have been extensively studied by classical immunohistochemical methods, particularly in the rat hypothalamus ${ }^{23,24,37,38}$ where they are synthetised together with neurophysins in the PVN, SON and accessory nuclei. ${ }^{22-24,37,39}$ Less data are available for mice, although this species is used increasingly frequently in neurobiology. ${ }^{40-46}$

By means of the iDISCO+ approach, we confirmed the neuronal localisation of both peptides in the SON and PVN. Alhough it was previously shown that this technique allows the double- and triple-labelling of neuronal cells, ${ }^{11,14-16}$ we did not conduct double staining of both peptides because primary antibodies were raised in the same species (rabbit). We also visualised the various accessory neurones previously identified in the rat anterior hypothalamus. ${ }^{23}$

iDISCO+ clearing revealed that the distribution of OXT and AVP neurones was not homogeneous along the SON at P5, as described previously in the rat. ${ }^{47,48}$ Both OXT and AVP immunoreactive cell bodies were found in the rostral part of the nucleus, whereas OXT neurones were more numerous in the dorsal part of the SON compared to AVP neurones. Classical immunohistochemical procedures on brain sections previously demonstrated that both peptide pathways not only project to the posterior pituitary, thus showing their well-known neuroendocrine effects, but also to some selective regions outside the hypothalamus, such as the amygdala and the nucleus accumbens. We also describe, for the first time, a dense population of AVP and OXT cell bodies and nerve fibres in the basoventral part of the hypothalamus, extending medially and ventrally, and in a rostral direction from the median eminence (Figure 5C,D; see Supporting information, Movies S3 and S4). Some of these cells may represent peptidergic neurones migrating from accessory nuclei towards SON and PVN.

Interestingly, these accessory neurones were more widespread at $\mathrm{P} 5$, in contrast to what has previously been reported in the adult rat hypothalamus. ${ }^{23}$ Some of these neurones are probably still migrating during this postnatal period to reach their final location in the adult brain. Furthermore, as shown in Figure 5 (see also Supporting information, Movies S3 and S4), the distribution of AVP neurones at P5 is very similar to that described in the adult rat PVN ${ }^{23}$ in contrast to OXT neurones. ${ }^{22}$ The OXT cell bodies, although more numerous in the PVN than AVP neurones at P5, were much smaller in volume than the AVP neurones. This could be linked to a delay in the maturation of the OXT system, as occurs in the rat brain, where AVP neurones have been reported to precede the development of OXT neurones that only become mature after birth. ${ }^{21,46,49-51}$

Late maturation of OXT neurones in the mouse was also supported by a progressive maturation of morphological and electrophysiological properties of OXT neurones. ${ }^{43}$ It was previously reported that neurophysin neurones showed dramatic changes in perikarial size during the mouse development in SON and PVN, with a plateau at P5 until P10, when a huge increase occured to reach adult values. ${ }^{40}$ The late maturation of OXT vs AVP neurones was also supported by long neuritic processes that can be observed at P5 in the PVN for AVP, whereas only very small processes were noted for OXT neurones (Figure 6 D,H).

The late maturation of OXT neurones compared to AVP- and $\mathrm{TH}$-expressing neurones may have functional significance. Both catecholaminergic and vasopressinergic systems are essential at birth for regulating plasma osmolarity, blood pressure, stress response and various metabolic effects via their release from nerve endings. By contrast, OXT effects following postnatal neurodevelopment need more time to become mature and functional. Such late maturation of hypothalamic OXT neurones may be linked to the acquisition of social skills later on in adulthood. ${ }^{23,43,45}$

Finally, the iDISCO+ method allowed us not only to quantify the number of AVP and OXT neuronal cell bodies, but also to measure their volume in a $3 \mathrm{D}$ representation. To our knowledge, there is no previous report available that addresses this issue. Quantitative methods in 3D neuronal regionalisation may thus be useful not only for providing information about the development of neurotransmitter/ neuropeptide systems, but also for quantifying the number/volume of 
such molecules in specific nuclei at different time points under various physiological and/or pathological conditions.

\section{5 | CONCLUSIONS}

The present study describes the potency of the improved clearing procedures coupled to novel image technologies with respect to better studying and anatomically visualising known or still unknown neurotransmitter substances involved in the brain and, more specifically in the present study, in hypothalamic neuroendocrine functions. This opens avenues for new research projects using various transgenic or wild-type mice, addressing important questions related to neuroanatomy and cerebral activity, such as migration, maturation processes and connectomic analysis. ${ }^{52}$ The findings may be applied when investigating various physiological and pathological situations, including, for example, catecholamine deficiency, sexual differenciation, lactation or dehydration. Such an imaging approach will provide exciting new information on the 3D distribution, morphology and projections of phenotypically-identified cells and pathways for future neuroanatomical and immunohistological studies.

\section{ACKNOWLEDGEMENTS}

We thank M. Belle and A. Chedotal for their help with the wholemount immunostaining protocols.

\section{ORCID}

W. Rostene iD http://orcid.org/0000-0003-0409-5361

\section{REFERENCES}

1. Björklund A, Hökfelt T. Classical neurotransmitters in the CNS. In: Björklund A, Hökfelt T, eds. Handbook of Chemical Neuroanatomy Part I. Amsterdam: Elsevier, 1984:1-463.

2. Björklund A, Hökfelt T. GABA and neuropeptides in the CNS. In: Björklund A, Hökfelt T, eds. Handbook of Chemical Neuroanatomy. Amsterdam, Elsevier, 1985:1-638.

3. Simmons DM, Swanson LW. High-resolution paraventricular nucleus serial section model constructed within a traditional rat brain atlas. Neurosci Lett. 2008;438:85-89.

4. Richardson DS, Lichtman JW. Clarifying tissue clearing. Cell. 2015;162:246-257.

5. Dobosz M, Ntziachristos V, Scheuer W, Strobel S. Multispectral fluorescence ultramicroscopy: three-dimensional visualization and automatic quantification of tumor morphology, drug penetration, and antiangiogenic treatment response. Neoplasia. 2014;16:1-13.

6. Dodt HU, Leischner U, Schierloh A, et al. Ultramicroscopy: threedimensional visualization of neuronal networks in the whole mouse brain. Nat Methods. 2007;4:331-336.

7. Keller PJ, Dodt HU. Light sheet microscopy of living or cleared specimens. Curr Opin Neurobiol. 2012;22:138-143.

8. Erturk A, Becker $\mathrm{K}$, Jahrling $\mathrm{N}$, et al. Three-dimensional imaging of solvent-cleared organs using 3DISCO. Nat Protoc. 2012; 7:1983-1995.

9. Erturk A, Lafkas D, Chalouni C. Imaging cleared intact biological systems at a cellular level by 3DISCO. J Vis Exp. 2014:89:1-12.
10. Renier N, Wu Z, Simon DJ, Yang J, Ariel P, Tessier-Lavigne M. iDISCO: a simple, rapid method to immunolabel large tissue samples for volume imaging. Cell. 2014;159:896-910.

11. Renier N, Adams EL, Kirst C, et al. Mapping of brain activity by automated volume analysis of immediate early genes. Cell. 2016;165: 1789-1802.

12. Pan C, Cai R, Quacquarelli FP, et al. Shrinkage-mediated imaging of entire organs and organisms using uDISCO. Nat Methods. 2016;13:859-867.

13. Belle $M$, Godefroy D, Dominici $C$, et al. A simple method for $3 D$ analysis of immunolabeled axonal tracts in a transparent nervous system. Cell Rep. 2014;9:1191-1201.

14. Launay PS, Godefroy D, Khabou H, et al. Combined 3DISCO clearing method, retrograde tracer and ultramicroscopy to map corneal neurons in a whole adult mouse trigeminal ganglion. Exp Eye Res. 2015;139:136-143.

15. Liebmann T, Renier N, Bettayeb K, Greengard P, Tessier-Lavigne M, Flajolet M. Three-dimensional study of Alzheimer's disease hallmarks using the iDISCO clearing method. Cell Rep. 2016;16:1138-1152.

16. Belle M, Godefroy D, Couly G, et al. Tridimensional visualization and analysis of early human development. Cell. 2017;169:161-173.

17. Soderblom C, Lee DH, Dawood A, et al. 3D imaging of axons in transparent spinal cords from rodents and nonhuman primates. eNeuro 2015;2(2). https://doi.org/10.1523/eneuro.0001-15.2015

18. Franklin KBJ, Paxinos G. The mouse brain in stereotaxic coordinates. San Diego, CA: Academic Press; 1997.

19. Anden NE, Dahlstrom A, Fuxe K, Larsson K. Mapping out of catecholamine and 5-hydroxytryptamine neurons innervating the telencephaIon and diencephalon. Life Sci. 1965;4:1275-1279.

20. Hökfelt T, Martensson R, Björklund A, et al. Distributional maps of tyrosine-hydroxylase-immunoreactive neurons in the rat brain. In: Björklund A, Hökfelt T, eds. Handbook of Chemical Neuroanatomy. Classical transmitters in the CNS, Part I. Amsterdam: Elsevier, 1984:277-379.

21. Buijs RM, Velis DN, Swaab DF. Ontogeny of vasopressin and oxytocin in the fetal rat: early vasopressinergic innervation of the fetal brain. Peptides. 1980;1:315-324.

22. Hawthorn J, Ang VT, Jenkins JS. Comparison of the distribution of oxytocin and vasopressin in the rat brain. Brain Res. 1984;307:289-294.

23. Ludwig M, Leng G. Dendritic peptide release and peptide-dependent behaviours. Nat Rev Neurosci. 2006;7:126-136.

24. Sofroniew MV. Vasopressin, oxytocin and their related neurophysins. In: Björklund A, Hökfelt T, eds. Handbook of Chemical Neuroanatomy. GABA and Neuropeptides in the CNS. Amsterdam: Elsevier 1985: 93-165.

25. Epp JR, Niibori Y, Liz HH, et al. Optimization of CLARITY for clearing whole-brain and other intact organs. eNeuro 2015;2(3). https://doi. org/10.1523/eneuro.0022-15.2015

26. Yeo SH, Kyle V, Morris PG, et al. Visualisation of Kiss1 neurone distribution using a Kiss1-CRE transgenic mouse. J Neuroendocrinol 2016; 28(11). doi.org/10.1111/jne.12435

27. Bjorklund A, Dunnett SB. Dopamine neuron systems in the brain: an update. Trends Neurosci. 2007;30:194-202.

28. Ugrumov M, Melnikova V, Ershov P, Balan I, Calas A. Tyrosine hydroxylase- and/or aromatic L-amino acid decarboxylase-expressing neurons in the rat arcuate nucleus: ontogenesis and functional significance. Psychoneuroendocrinology. 2002;27:533-548.

29. Balan IS, Ugrumov MV, Calas A, Mailly P, Krieger M, Thibault J. Tyrosine hydroxylase-expressing and/or aromatic L-amino acid decarboxylase-expressing neurons in the mediobasal hypothalamus of perinatal rats: differentiation and sexual dimorphism. J Comp Neurol. 2000;425:167-176.

30. Sawchenko PE, Swanson LW. The organization of noradrenergic pathways from the brainstem to the paraventricular and supraoptic nuclei in the rat. Brain Res. 1982;257:275-325. 
31. Ugrumov MV, Tixier-Vidal A, Taxi J, Thibault J, Mitskevich MS. Ontogenesis of tyrosine hydroxylase-immunopositive structures in the rat hypothalamus. Fiber pathways and terminal fields. Neuroscience. 1989;29:157-166.

32. Abramova M, Marsais F, Calas A, Thibault J, Ugrumov M. Dynamical study of tyrosine hydroxylase expression and its correlation with vasopressin turnover in the magnocellular neurons of the supraopticoposthypophysial system under long-term salt loading of adult rats. Brain Res. 2002;925:67-75.

33. Abramova MA, Calas A, Ugrumov M. Vasopressinergic neurons of the supraoptic nucleus in perinatal rats: reaction to osmotic stimulation and its regulation. Brain Struct Funct. 2011;215:195-207.

34. Ugrumov MV, Popov AP, Vladimirov SV, Kasmambetova S, Thibault J. Development of the suprachiasmatic nucleus in rats during ontogenesis: tyrosine hydroxylase immunopositive cell bodies and fibers. Neuroscience. 1994;58:151-160.

35. Beltramo M, Calas A, Chernigovskaya E, et al. Postnatal development of the suprachiasmatic nucleus in the rat. Morpho-functional characteristics and time course of tyrosine hydroxylase immunopositive fibers. Neuroscience. 1994;63:603-610.

36. Wilson CA, Dakin CL, Rico JA, Golmohamad A, Ahmad-Jauhari Y, Davies DC. The anti-dopaminergic agent, haloperidol, antagonises the feminising effect of neonatal serotonin on sexually dimorphic hypothalamic nuclei and tyrosine hydroxylase immunoreactive neurones. J Neuroendocrinol. 2009;21:648-656.

37. Sofroniew MV, Weindl A. Projections from the parvocellular vasopressin- and neurophysin-containing neurons of the suprachiasmatic nucleus. Am J Anat. 1978;153:391-429.

38. Buijs RM. The development of vasopressin and oxytocin systems in the brain. In: Björklund A, Hökfelt A, Tohyama M, eds. Ontogeny of transmitters and peptides in the CNS. Amsterdam: Elsevier, 1992:547-572.

39. Vandesande F, Dierickx K. Identification of the vasopressin producing and of the oxytocin producing neurons in the hypothalamic magnocellular neurosecretroy system of the rat. Cell Tissue Res. 1975;164:153-162.

40. Silverman AJ, Goldstein R, Gadde CA. The ontogenesis of neurophysincontaining neurons in the mouse hypothalamus. Peptides. 1980;1:27-44.

41. Castel M, Morris JF. The neurophysin-containing innervation of the forebrain of the mouse. Neuroscience. 1988;24:937-966.

42. Castel M, Feinstein N, Cohen S, Harari N. Vasopressinergic innervation of the mouse suprachiasmatic nucleus: an immuno-electron microscopic analysis. J Comp Neurol. 1990;298:172-187.

43. Grinevich V, Desarmenien MG, Chini B, et al. Ontogenesis of oxytocin pathways in the mammalian brain: late maturation and psychosocial disorders. Front Neuroanat. 2014;8:164.
44. Rood BD, De Vries GJ. Vasopressin innervation of the mouse (Mus musculus) brain and spinal cord. J Comp Neurol. 2011;519:2434-2474.

45. Otero-Garcia M, Agustin-Pavon C, Lanuza E, Martinez-Garcia F. Distribution of oxytocin and co-localization with arginine vasopressin in the brain of mice. Brain Struct Funct. 2016;221:3445-3473.

46. Ugrumov MV. Magnocellular vasopressin system in ontogenesis: development and regulation. Microsc Res Tech. 2002;56:164-171.

47. Hou-Yu A, Lamme AT, Zimmerman EA, Silverman AJ. Comparative distribution of vasopressin and oxytocin neurons in the rat brain using a double-label procedure. Neuroendocrinology. 1986;44: 235-246.

48. Callewaere C, Banisadr G, Desarmenien MG, et al. The chemokine SDF-1/CXCL12 modulates the firing pattern of vasopressin neurons and counteracts induced vasopressin release through CXCR4. Proc Natl Acad Sci USA. 2006;103:8221-8226.

49. Jing $X$, Ratty AK, Murphy D. Ontogeny of the vasopressin and oxytocin RNAs in the mouse hypothalamus. Neurosci Res. 1998;30:343-349.

50. Trembleau A, Ugrumov M, Roche D, Calas A. Vasopressin and oxytocin gene expression in intact rats and under catecholamine deficiency during ontogenesis. Brain Res Bull. 1995;37:437-448.

51. Hyodo S, Yamada C, Takezawa T, Urano A. Expression of provasopressin gene during ontogeny in the hypothalamus of developing mice. Neuroscience. 1992;46:241-250.

52. Cazemier JL, Clasca F, Tiesinga PH. Connectomic analysis of brain networks: novel techniques and future directions. Front Neuroanat. 2016;10:110.

\section{SUPPORTING INFORMATION}

Additional Supporting Information may be found online in the supporting information tab for this article.

How to cite this article: Godefroy D, Dominici C, Hardin-Pouzet $\mathrm{H}$, et al. Three-dimensional distribution of tyrosine hydroxylase, vasopressin and oxytocin neurones in the transparent postnatal mouse brain. J Neuroendocrinol. 2017;29:e12551. https://doi.org/10.1111/jne.12551 